\title{
Enceladus as a place of origin of life in the Solar System
}

\author{
Leszek CZECHOWSKI ${ }^{1, *}$ \\ 1 University of Warsaw, Faculty of Physics, Institute of Geophysics, Pasteura 5, 02-093 Warszawa, Poland
}

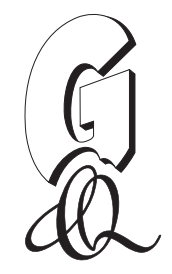

Czechowski, L., 2018. Enceladus as a place of origin of life in the Solar System. Geological Quarterly, 62 (1): 172-180, doi: 10.7306/gq. 1401

Enceladus, a satellite of Saturn, with its radius of $250 \mathrm{~km}$, is the smallest geologically active celestial body in the Solar System. My model of core origin and evolution indicates that for hundreds of My after accretion Enceladus was an appropriate body for an origin of life. I continue consideration of the hypothesis that Enceladus was a cradle of life in the Solar System. I found that simple organisms could be ejected in icy grains into the space by volcanic jets or by meteoroid impacts. Several mechanisms could be responsible for later transport of the grains to the early Earth and other terrestrial planets. Eventually I suggest that Enceladus is the most appropriate body for a cradle of life in the Solar System.

Key words: life origin, Enceladus, panspermia, gravity assist, Poynting-Robertson effect, impact.

\section{INTRODUCTION}

In the Saturnian system there are two bodies on which the conditions allow for the existence of biological life. These are Titan and Enceladus. Titan is the only body in the Solar System, other than Earth, that has stable liquids (hydrocarbons and nitrogen) on its surface. McKay (2016) considered the possibility of carbon-based organisms living in Titan liquids. He indicated that photochemically produced acetylene could be a source of biological energy in addition to photosynthesis.

Czechowski $(2014 a$, b) has noted that the temperature inside Enceladus is rather moderate and allows for the existence of liquid water. Moreover he indicated a possible source of energy for primitive organisms and potential ways of their proliferation. In the present paper I consider the hypothesis that Enceladus is a cradle of life for the Solar System.

The idea of panspermia is usually attributed to Arrhenius (1908). However, the beginning of the hypothesis and the term itself are much older. The Greek philosopher Anaxagoras ( 510 BC- 428 BC) was a precursor (O'Leary, 2008). In the 19 th century the hypothesis was proposed by Thomson (1871) and others.

In general, the hypothesis of panspermia states that living organisms can be transported through the space from one celestial body to another. In this way, life may be a common phenomenon on many celestial bodies even if it has originated only on one of them. Panspermia assumes that, at least once, life originated in the Universe as a result of the natural processes

\section{*E-mail: Iczech@op.pl}

Received: August 12, 2017; accepted: October 31, 2017; first published online: February 7, 2018 (abiogenesis) but does not address the problem how this original life began.

There are a few versions of the hypothesis. In the present paper I concentrate on the idea that microorganisms or their endospores are transported within larger solid grains or in comets that protect the organisms from lethal radiation. If organisms meet proper conditions on a new body's surface, they become active. The later process of evolution leads to origin of new species, eventually forming global ecosystems specific for the planet.

Most presently discussed hypotheses concerning the origin of life assumes that life on the Earth is of terrestrial origin. Indeed, the more primitive bacteria (or their fossils) we find on Earth, the more we reduce the need for panspermia. Microfossils from Hudson Bay (Dodd et al., 2017) support the hypothesis that hydrothermal vents may have been the cradle of life on Earth. Note, however, also that the suggestion of "an almost instantaneous emergence of life" after ocean formation $4.41 \mathrm{Ga}$ (see Dunham, 2017) can be interpreted as reflecting the earlier existence of biological life, which then used the first opportunity to colonize the terrestrial oceans. Such an interpretation supports the idea of panspermia. Note also some indications that terrestrial organisms are a result of evolution longer than the age of the Solar System - e.g., Sharov and Gordon (2013). If this hypothesis were correct then panspermia would be a necessary process for the presence of life on the Earth.

Several mechanisms responsible for panspermia have been suggested. The simplest one is the transport of organisms in the ejecta resulting from an impact of a large meteoroid. The Martian meteorites found on Earth indicate that ejecta generated by an impact could reach a velocity exceeding $5 \mathrm{~km} \mathrm{~s}^{-1}$ (the escape velocity from Mars is $5.027 \mathrm{~km} \mathrm{~s}^{-1}$ ).

Enceladus is a medium-sized icy satellite (MIS) of Saturn. MIS are built of mixture of rocks (i.e. non-volatiles probably of chondrite composition) and ices. Enceladus with its radius of 


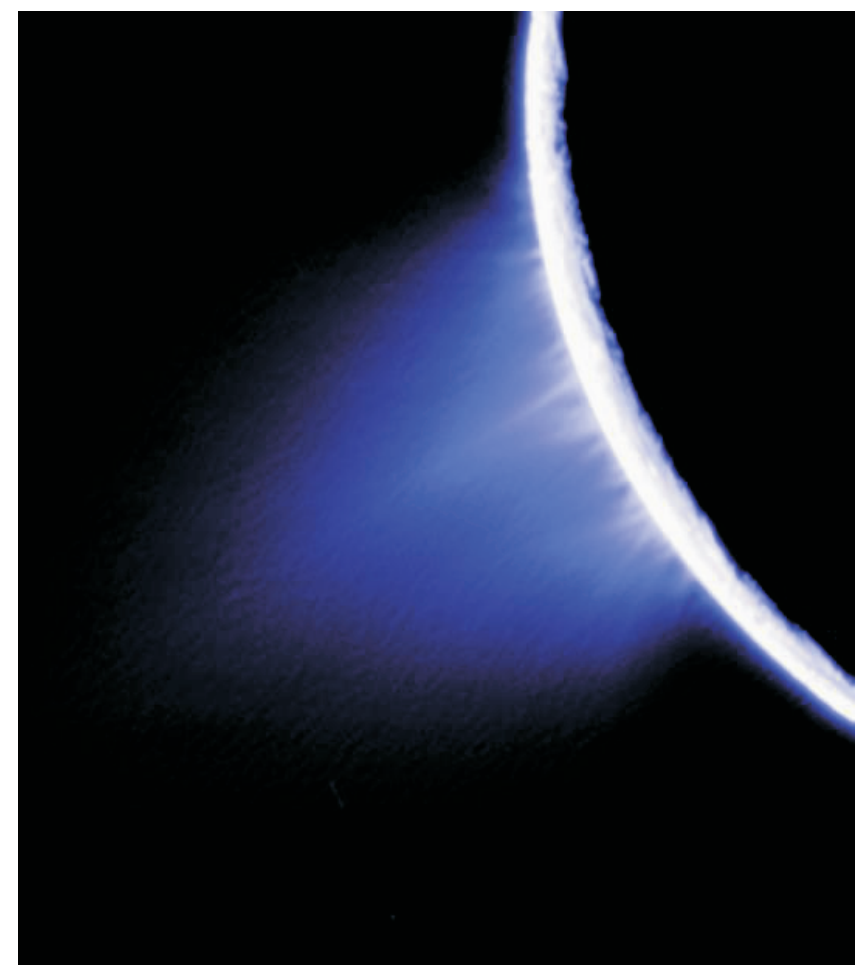

Fig. 1. The cryovolcanic activity of Enceladus transports grains from the core to E-ring

Image presents volcanic jets in the southern hemisphere of Enceladus. Picture taken by Cassini (on Nov. 27th, 2005) and processed to reveal the individual jets (image credit NASA/JPL/Space Science Institute, after https://photojournal.jpl.nasa.gov/jpegMod/PIA08386_modest.jpg, retrieved Feb. 5, 2018)

$250 \mathrm{~km}$ is one of the smallest of MIS, however, contrary to the most of them, it is geologically active (Figs. 1 and 2).

Cryovolcanic activity on Enceladus is concentrated along the tiger stripes, i.e. along a series of sub-parallel, linear depressions (grooves) flanked on each side by low ridges. The official term is sulcus (plural sulci) while the given proper names are Alexandria Sulcus, Cairo Sulcus, Baghdad Sulcus and Damascus Sulcus. They are spaced $\sim 35$ kilometres apart. Each tiger stripe is $\sim 130 \mathrm{~km}$ long, $\sim 2 \mathrm{~km}$ wide, and $\sim 0.5 \mathrm{~km}$ deep. Their temperature is higher at $45-89 \mathrm{~K}$ than the expected temperature for this region of Enceladus if the region were heated solely from sunlight.

Tiger stripes are probably of tectonic origin. However, their correlation with warm regions and large jets (geysers) suggests that they could be fissures in this moon's lithosphere. Data from Cassini indicate complex organic material in jets ejected from the satellite. Over 100 geysers have been identified.

Geysers located within the tiger stripes emanate water vapor, ice grains, $\mathrm{CO}_{2}$, nitrogen, $\mathrm{CH}_{4}$ and other organics (e.g., Fig. 3; Porco et al., 2006; Waite et al., 2009). The jets are probably generated from a near-surface body of liquid water (e.g., Hargitai and Kereszturi, 2015). Geyser activity is correlated with deformations of the satellite by tidal forces of Saturn. Under these stresses, the tiger stripes might open and close at different times (e.g., Kohler, 2015). The combinations of these motions may play a significant role in determining when the geysers are most active. The surface along the tiger stripes is generally smooth and young (from the low number of impact craters). It is covered probably by back-fallen material.

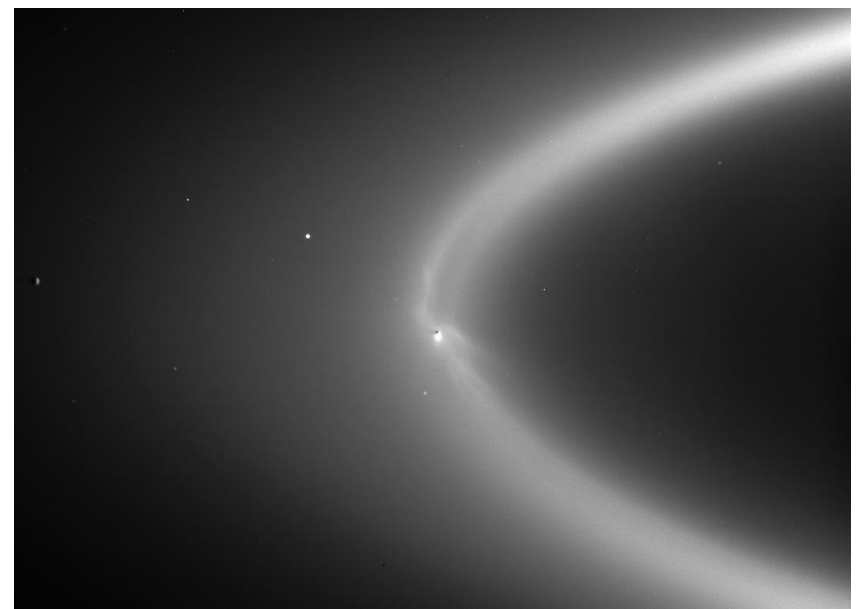

Fig. 2. Enceladus moving in the E-ring

The E-ring consists of particles ejected from Enceladus (NASA from https://photojournal.jpl.nasa.gov/catalog/?IDNumber=PIA08321 retrieved Feb. 5, 2018)

The cryovolcanic activity of Enceladus is responsible for transport of grains from the core to the E-ring of Saturn. Possible organisms (in a dormant phase) might be also transported within these grains.

A recent paper of Ćuk et al. (2016) suggests that Enceladus was formed $\sim 100 \mathrm{Ma}$. The hypothesis that Enceladus was a cradle of the Solar System requires the existence of the satellite $\sim 3.8 \mathrm{Ga}$ (i.e. the age of the oldest terrestrial fossils). The "traditional" age of Enceladus is assumed here (e.g., Mousis et al., 2009) instead of Ćuk et al. (2016). However, if the present paper considers the general possibility of transfer of genetic material from the present Enceladus to the terrestrial planets, the age of the satellite is not critical.

Orbital changes during the first $30 \mathrm{Ma}$ after formation of the Solar System are not critical for the hypothesis (cf. e.g., Batygin and Brown, 2010). Czechowski (2014a) found that for $200 \mathrm{Ma}$

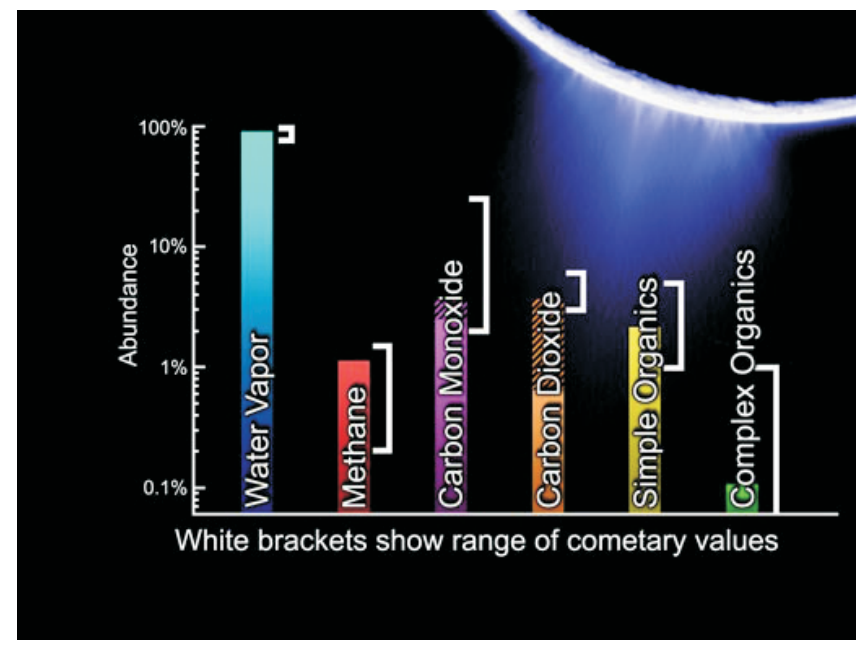

Fig. 3. Comparison of chemical composition of Enceladus jets and cometary material

Note high content of organic material including complex organic (Image credit: NASA/JPL/SwRI, https://photojournal.jpl.nasa.gov/catalog/?IDNumber=PIA10357, retrieved Feb. 5, 2018) 
the evolution of Enceladus was determined by short-living radioactive isotopes and the tidal heating is not necessary.

\section{CONDITIONS IN THE CORE OF ENCELADUS}

Czechowski (2014a) considered the process of differentiation and core formation in Enceladus. He found that the result of differentiation is a relatively cold core of loosely packed grains with liquid water between them (cf. the models of Schubert et al., 2007; Malamud and Prialnik, 2013). The liquid water and silicates make the process of serpentinization possible. After Abramov and Mojzsis (2011) the reaction of serpentinization may be:

$\mathrm{Mg}_{2} \mathrm{SiO}_{4}$ (forsterite) $+\mathrm{MgSiO}_{3}$ (enstatite) $+2 \mathrm{H}_{2} \mathrm{O} \rightarrow$ $\mathrm{Mg}_{3} \mathrm{Si}_{2} \mathrm{O}_{5}(\mathrm{OH})_{4}$ (antigorite).

This reaction releases $241000 \mathrm{~J}$ per $1 \mathrm{~kg}$ of serpentine produced.

The density of Enceladus indicates that the present mass fraction of silicates $f_{\text {mas }}$ is $\sim 0.646$, hence the total mass of its silicates is $\sim 6.97 \times 10^{19} \mathrm{~kg}$. Approximately $80 \%$ of these could react with water (Czechowski and Losiak, 2016), amounting to $\sim 5.6 \times 10^{19} \mathrm{~kg}$. Serpentinisation is believed to be a possible source of energy for primitive life (e.g., Russell et al. 2010; Nitschke and Russell, 2010; Góbi and Kereszturi, 2017). Moreover the reaction also influences planetary geological evolution. According to Russell et al. (2010): "For life to have emerged [...], a sustained source of chemically transducible energy was essential. The serpentinization process is emerging as an increasingly likely source of that energy. Serpentinization of ultramafic crust would have continuously supplied hydrogen, methane, [...] to off-ridge alkaline hydrothermal springs that interfaced with the metal-rich carbonic Hadean Ocean"; see also Izawa et al. (2010) and Weiss et al. (2016).

The results of Dodd et al. (2017) emphasized the role of serpentinization for the origin of life. They found that the probable genome of the Last Universal Common Ancestor (LUCA) of all terrestrial organisms had "[...] 355 genes pointed quite precisely to an organism that lived in the conditions found in deep sea vents $[\ldots] "$.

The pressure in the centre of Enceladus is $\sim 2.3 \times 10^{7} \mathrm{~Pa}$ which corresponds to the pressure at the depth of $2300 \mathrm{~m}$ in terrestrial oceans.

The highest possible temperature in the early Enceladus is given in Figure 4. In this calculation the transport of heat is neglected, so no heat leaves the satellite; therefore, the result of calculation does not depend on particulars of convection or conduction. The temperature depends only on the time of the beginning of accretion $t_{\text {ini }}$ and on the fraction $f_{\text {mas }}$ (for details, see similar calculation of Czechowski, 2014a). The earlier accretion means more intense heating by short-lived isotopes. Note, that even for very early accretion, liquid $\mathrm{H}_{2} \mathrm{O}$ may exist in the core and the temperature does not exceed the temperature required for living organisms (e.g., Clarke, 2014). Other calculations of Czechowski (2014a) indicated that such conditions could have lasted for a few hundreds of My in the interior of Enceladus. Presently, tidal heating keeps the Enceladus interior warm.

The main conclusion of Czechowski $(2014 a, b)$ that the interior of Enceladus was favourable for the life, was supported by Hsu et al. (2015).

Since terrestrial rocks are permeable up to the pressure of $\sim 300 \mathrm{MPa}$ then one can expect that the silicates in the core of Enceladus were also permeable for liquids and gases. This could have led to the formation of extensive hydrothermal convective systems allowing water to contact with most of the silicates. Hence, in Enceladus most of the reactive silicates may be serpentenized. Its total mass (i.e. $\sim 5.6 \times 10^{19} \mathrm{~kg}-$ see

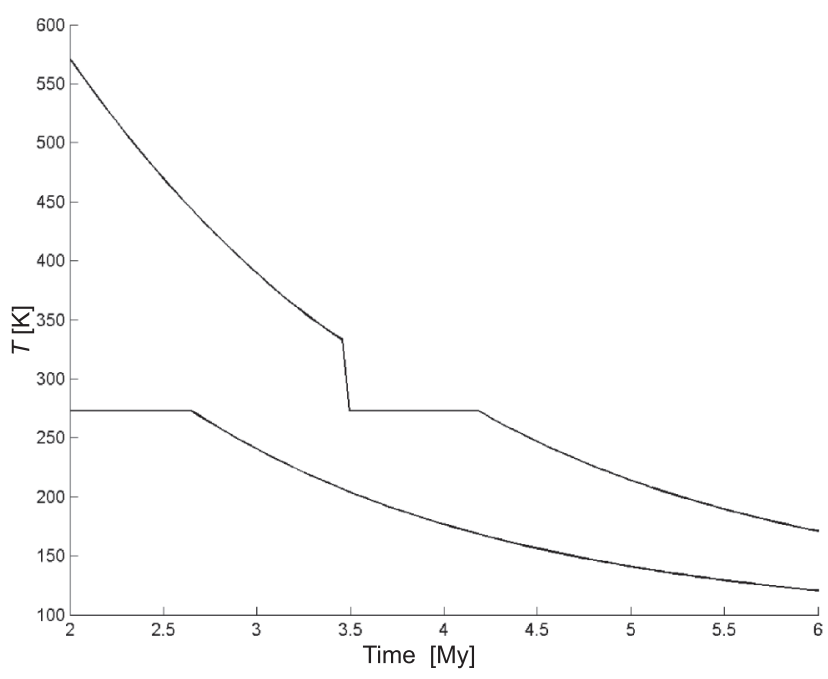

Fig. 4. Graph of the potential temperature for a satellite with $f_{\text {mas }}=0.281$ (Mimas or proto-Enceladus, the lower line) and for $f_{\text {mas }}=\mathbf{0 . 6 4 6}$ (present Enceladus, the upper line) versus time of beginning of accretion $t_{\mathrm{ini}}$

The heat transfer is neglected, so all heat is used for the temperature increase and the melting. The heat is a result of the radioactive decay during $20 \mathrm{My}$ (after time $t_{\text {ini }}$ ) and the serpentinization. The effect of serpentinization is seen as a sharp step on the upper line. Note large difference between the potential temperature for present Enceladus and proto-Enceladus

above) distributed uniformly over the area of Earth gives $\sim 110$ ton per $1 \mathrm{~m}^{2}$. Note that in the Earth only minor proportion of silicates have contact with water (e.g., in neovolcanic zones at spreading centres). This could means that total mass of serpentenized silicates in Enceladus might be even comparable to that in the Earth.

To conclude: for a long time ( $400 \mathrm{Ma})$ conditions in the core of Enceladus were favourable for the origin and preservation of biological life. There were liquid water, organic compounds, moderate temperature, a lack of energetic radiation, and a source of chemical energy. This fits well to an environment for the LUCA (e.g., Brasier, 2012). Of course, perfectly constant conditions do not necessarily help to develop life. Oceanic tides, lightning, and specific conditions in shallow-water are mentioned as factors helpful for developing the complicated, self-replicating molecules necessary for life. Analogous effects were and are present in the core of Enceladus. Regular tidal deformations and/or thermal convection may have given rise to transport of molecules, helping to develop more complex molecules.

\section{PROLIFERATION OF THE LIFE}

Let us now consider some mechanisms for spreading life to other celestial bodies. These depend on the specific conditions on the parental body.

According to Yang et al. (2009) microorganisms in the terrestrial atmosphere are found as far as $77 \mathrm{~km}$ above the surface. However, bare organisms are not protected against cosmic radiation. Such protection could be given by solid grains. Probably, a large impact is the only realistic mechanism that could eject solid grains from a terrestrial planet at a speed large enough to reach another planet. The Martian meteorites found on Earth proves that this mechanism works for Mars. For the Earth it is less probable because of a high escape velocity and dense atmosphere. 
There are a few possible mechanisms of transport of organisms from Enceladus to other planets. Their stages are discussed in a next few subsections.

FROM THE CORE TO THE SURFACE

The temperature, the pressure and strong radiation from the magnetosphere of Saturn at the surface of Enceladus do not allow the existence of unprotected life. Life could exist only at the depth of several kilometres below the surface. So, the first step for the proliferation of life is reaching the surface of the satellite. This problem does not exist for terrestrial life because it originated on an oceanic floor and oceanic currents could transfer organisms to the water's surface. However, almost continuous and repeated cryovolcanic activity is observed at the polar region of Enceladus. This could transport matter in the jets of gas and solid/frozen particles from the interior (see the next subsection). The ancient forms of this activity may have been similar to the present forms. The organisms could be frozen into and transported within icy grains. Note that the temperature of the cryovolcanic gases is rather low, so it would not kill the organisms. Note also that the possible presence of ammonia additionally decreasing the melting point of $\mathrm{H}_{2} \mathrm{O}$.

\section{FROM THE SURFACE TO SPACE}

The existence of an E-ring is evidence that cryovolcanic jets could eject gas and solid particles (possibly with primitive organisms) into orbit around Saturn (Figs. 1-3). Most grains ejected into space are of micrometre scale, however ejection of boulders is also possible especially in periods of enhanced volcanic activity. To investigate the problem we developed a model of acceleration of grains in the volcanic pipe in Enceladus.

In particular nozzles (de Laval nozzles) the expanding gas could reach supersonic speed. However, a more realistic assumption is that the velocity of gas in the volcanic pipe does not exceed the speed of sound. Assuming a density of water

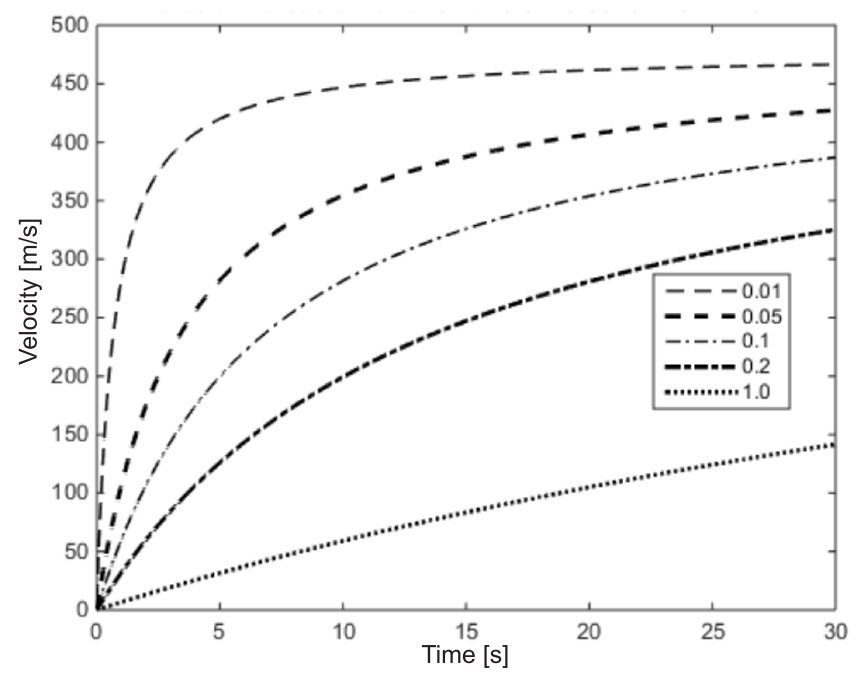

Fig. 5. Velocity of grains versus time in the volcanic pipe

The grains sizes are $1 \mathrm{~cm}$ (the uppermost line), $5 \mathrm{~cm}, 10 \mathrm{~cm}, 20 \mathrm{~cm}$, $1 \mathrm{~m}$ (the lowermost line). The density of grains is $500 \mathrm{~kg} \mathrm{~m}^{-3}$, drag coefficient $C_{D}=2$, gas velocity is equal to the speed of sound (i.e. $478 \mathrm{~m} \mathrm{~s}^{-1}$ ) vapour of $0.01 \mathrm{~kg} \mathrm{~m}^{-3}$ and a velocity of expansion in the volcanic pipe of $478 \mathrm{~m} \mathrm{~s}^{-1}$ (sound velocity) we found that even grains of $20 \mathrm{~cm}$ diameter could reach escape velocity (i.e. $239 \mathrm{~m} \mathrm{~s}^{-2}$ for Enceladus) in pipes a few km long (Fig. 5). Larger grains could also be ejected if the moving gas has higher density or moves along longer pipes.

The particles forming the E-ring orbit Saturn. Particles of lower velocity could reach an orbit around Enceladus but not an orbit around Saturn. I do not consider these slower particles here.

The situation on the Earth is different. Terrestrial volcanic activity cannot eject rocks into orbit because the speed of sound is significantly lower ( 20 times lower) than the orbital velocity of a low-orbit satellite $\left(\sim 8 \mathrm{~km} \mathrm{~s}^{-1}\right)$ and lower than the escape velocity $\left(\sim 11.2 \mathrm{~km} \mathrm{~s}^{-1}\right)$. Moreover drag in the terrestrial atmosphere additionally reduces the velocity of the volcanic ejecta.

\section{FROM THE E-RING TO A HELIOCENTRIC ORBIT}

To reach a heliocentric orbit, the grains on the orbit inside the E-ring must be accelerated to the escape velocity from Saturn. This requires $\sim 5 \mathrm{~km} \mathrm{~s}^{-1}$ speed increase (depending on the orbit in the E-ring). Gravity assistance could be responsible for this acceleration.

Gravity assistance is an effect of gravitational interaction of a small body (e.g., a spacecraft) with a planet (or a satellite). Its result is similar to an elastic collision with a moving planet, i.e. the spacecraft changes its velocity depending on the orbital velocity of the planet. This was used in many interplanetary missions to reach velocity change without losing fuel. The main problem is that spacecraft trajectory is carefully chosen to reach the required effect while the trajectory of the grain is determined by accidental initial velocity.

The existence of several satellites of Saturn increases the probability of effective gravity interactions and the acceleration of some grains. Sequence of close encounters could eventually transfer enough energy to some grains to allow them to change their orbit around Saturn into a heliocentric orbit.

The mechanism of gravity assistance may be an important factor at two stages: (1) for acceleration of particles from orbit around Saturn into orbit around the Sun and (2) for deceleration of the particle at the next step, i.e. from a heliocentric orbit close to the orbit of Saturn to an orbit close to terrestrial planets. The main advantage of this mechanism is that it works for bodies large enough to be a shelter against cosmic radiation.

To investigate this effect I developed a 3D numerical model of the Saturnian System (the planet, the largest satellite Titan and six medium-sized satellites are included). In numerical experiments I investigated the trajectories and energy of grains ejected from Enceladus with different velocities in various, randomly chosen, directions. The initial velocity is high enough to allow the grain to reach orbits in the E-ring. Many of them could reach the orbit of Titan, the gravity of which could be an important factor in the gravity assistance.

I performed $\sim 400$ such experiments. A substantial increase in the velocity (of $\sim 1 \mathrm{~km} \mathrm{~s}^{-1}$ ) was found in $\sim 2 \%$ cases. However, in none of these experiments did the grain reach enough energy to leave the system of Saturn. This indicates that the probability of leaving the system as a result of this mechanism is low. However, over millions of years the number of grains or larger boulders ejected into space could be many orders higher than the number of my simulations, so some ejecta might reach heliocentric orbit. For successful proliferation, the ejection even of a small number of boulders with living organisms could be sufficient. 
DECELERATION OF THE PARTICLES DUE

TO THE POYNTING-ROBERTSON EFFECT

To reach the terrestrial planets from a heliocentric orbit close to Saturn the grain must be substantially decelerated. There are a few possible mechanisms of such loss of energy and momentum: the Poynting-Robertson mechanism (for grains larger than a few $\mu \mathrm{m}$ ), the Yarkovsky diurnal effect (if the grain is a retrograde rotator) and Yarkovsky seasonal effect (for grains a few metres across); e.g., Pater and Lissauer (2001), and gravity assistance. Deceleration leads the particle to move closer to Earth and to other terrestrial planets.

Consider the Poynting-Robertson effect which is effective for typical grain sizes of E-ring particles. It is a result of photon-grain interaction. Assume a grain on a circular orbit with radius $R$ and photons radially emitted by the Sun. In a grain's frame of reference the photons have some tangent component of velocity. This gives rise to a tangential force opposite to the velocity of the grain (e.g., Guess, 1962). This force is given by the formula:

$$
F_{P R}=\frac{v W}{c^{2}}
$$

where: $v$ - the orbital velocity of the grain, $W$ - the power of Sun's radiation, $c-$ the speed of light.

The power of drag is:

$$
P_{\text {drag }}=F_{P R} V=v^{2} \frac{D}{(R c)^{2}}
$$

Note that $D=C_{\text {SunE }} S_{g r}\left(R_{E}\right)^{2}$, where $C_{\text {SUnE }}=1350 \mathrm{~W} \mathrm{~m}^{-2}$ is the Solar constant at the Earth orbit, $S_{g r}=\pi r_{g r}^{2}$ is the cross-section of the grain, $r_{g r}$ is the grain radius and $R_{E}$ is the radius of the Earth's orbit. The orbital energy of the grain is given by:

$$
E_{o r b}=\frac{G M m}{R}+\frac{1}{2} m v^{2}
$$

where: $M$ - the mass of Sun, $m$ - the mass of the grain and the velocity of the grain is: $v=(G M / R)^{1 / 2}$.

Substituting $v$ one can obtain:

$$
E_{\text {orb }}=\frac{G M m}{2 R}
$$

Differentiation of $E_{\text {orb }}$ with respect to time gives:

$$
\frac{d E_{o r b}}{d t}=\frac{G M m}{2 R^{2}} \frac{d R}{d t}
$$

Comparison of $d E_{o r b} / d t$ and $P_{d r a g}$ and integration indicate that the time of falling from an initial orbit with radius $R_{i}$ to an orbit with radius $R_{E}$ is given by:

$$
t=\frac{m c^{2}}{4 D}\left(R_{i}^{2}-R_{E}^{2}\right)
$$



Fig. 6. Time of falling from the orbit with the radius $9.5 \mathrm{AU}$ to a given orbit as a result of Poynting-Robertson effect

The grains density is assumed to be $1000 \mathrm{~kg} \mathrm{~m}^{-3}$. The values of grains' radius are: $5 \mu \mathrm{m}, 10 \mu \mathrm{m}, 15 \mu \mathrm{m}, 20 \mu \mathrm{m}, 25 \mu \mathrm{m}$ from the lowest line to the uppermost line, respectively

Figure 6 shows the time of falling from an orbit with a radius 9.5 AU to a given orbit as a result of the Poynting-Robertson effect. The grain density is assumed to be $1000 \mathrm{~kg} \mathrm{~m}^{-3}$. For a grain radius of $10 \mu \mathrm{m}$ the time of reaching Earth's orbit from the Saturnian one is $\sim 650 \mathrm{ka}$. This result is not especially encouraging because of the long duration and the small size of the grain. Such grain could not protect organisms against radiation for such long time. Note that for large grains (e.g., $\sim 1 \mathrm{~m}$ across) other processes, like the Yarkovsky effect, could be more effective than the Poynting-Robertson effect. Unfortunately, the effects of those mechanisms are much slower than those discussed in previous and subsequent subsections, consequently they are of lower significance for panspermia.

It is suggested by one of manuscript reviewers (Dr. Chris McKay) that the above discussion does not exclude small particles (e.g., $\sim 10 \mu \mathrm{m}$ ) as a possible medium of transport. He indicated that small grains are more common in Enceladus's jets than larger ones and they could give, at least for some time, protection against solar UV. In space, they could be easily picked up by comets and be protected against cosmic radiation within the comet. In fact, comets would be the fastest means of transport of microorganisms from Saturn to the terrestrial planets.

\section{LARGE IMPACTS AS MECHANISM OF PANSPERMIA}

A large impact of meteoroid seems to be a silver bullet for many problems of panspermia. Catastrophic collision like that of proto-Earth and Theia could eject into heliocentric orbit rocks from a large terrestrial planet. Smaller, but still large impacts were probably responsible for producing the Martian meteorites found on the Earth (e.g., the SNC groups of meteorites). This means that large impacts could produce ejecta with a velocity exceeding the escape velocity from Mars, i.e. $5.027 \mathrm{~km} \mathrm{~s}^{-1}$. In fact, the initial ejecta velocity was probably significantly higher because in the ancient Martian atmosphere (more dense than the present one) the ejecta were subject to significant drag. 
The very rare atmosphere of Enceladus cannot significantly reduce the velocity of ejecta. If a large object impacts the icy crust of Enceladus and ejects into space grains (boulders) with a velocity $>5 \mathrm{~km} \mathrm{~s}^{-1}$ then some of them leave the Saturnian System and could reach the trajectory leading to terrestrial planets. This depends only on the direction of the velocity. Other mechanisms, like those discussed above, were not necessary in such a case. The elastic modulus of water ice is significantly lower (approximately one order lower) than the modulus for silicates (e.g., Nimmo, 2004; Pabst and Gregorová, 2013). Fortunately, the lower density of ice makes the acceleration of ice grains by $5 \mathrm{~km} \mathrm{~s}^{-1}$ still possible.

The impact ejecta contain rocks from the surface down to a depth equal to $\sim 20 \%$ of the radius of the crater (e.g., Melosh, 2011), so a large impact could reach the depth where the life flourishes. Moreover, surface material ejected by geysers could also contain dormant microorganisms within grains.

Many ejected grains will be shocked and crushed (see e.g., Bryce et al., 2014). The strength of water ice is lower than that of most silicates. In some ejected grains the shock could result in significant temperature increase and evaporation of the grains. The most shocked rocks are close to the impacting body. However, the pressure of the shock decreases with the distance and consequently at some distance from the crater the matter is subject to moderate increase in temperature only. To accelerate a mass of $1 \mathrm{~kg}$ (e.g., a cube of size $\sim 0.1 \mathrm{~m}$ ) to $5 \mathrm{~km} \mathrm{~s}^{-1}$ in 0.1 second requires a force of $50 \mathrm{kN}$. This force acting on a surface of area of $0.01 \mathrm{~m}^{2}$ (one side of the cube) corresponds to a pressure of $5 \mathrm{MPa}$ and results in only moderate temperature increase.

For instance, in the well-known meteorite ALH8400 found in Antarctica in 1984, there are no obvious signs of the shock and even small structures are well preserved. A few of those structures were considered by some scientists as fossil organisms (e.g., McKay et al., 1996; Borg et al., 1999).

\section{TIME OF FALLING TO TERRESTRIAL ORBIT}

Let us assume that the grain containing some organisms is ejected outside Saturn's system as a result of impact or of gravitational assistance. If grains have a velocity higher than Saturn (with respect to the Sun) then they will move away from the Sun. If the grains' velocity is lower than the orbital velocity of Saturn, they will move closer to the Sun. Assume that the grain has negligible velocity with respect to the Sun and consider the process of free falling of the grain toward the orbits of the terrestrial planets as a result of the Sun's gravity. According to the law of energy conservation the kinetic energy $E_{k}$ is the difference between the current potential gravitational energy $E_{P}$ and the initial energy $E_{P O}$.

$$
E_{k}=\frac{1}{2} m\left(\frac{d r}{d t}\right)^{2}=E_{P}-E_{P 0}
$$

where: $r$ - the distance of the grain from the Sun.

Assuming that the mass of the grain is $m=1 \mathrm{~kg}$ then the velocity $d r / d t$ is equal to:

$$
\frac{d r}{d t}=\sqrt{2 G M\left(\frac{1}{R_{0}}-\frac{1}{r}\right)}=\sqrt{2 G M\left(\frac{r-R_{0}}{R_{0} r}\right)}
$$

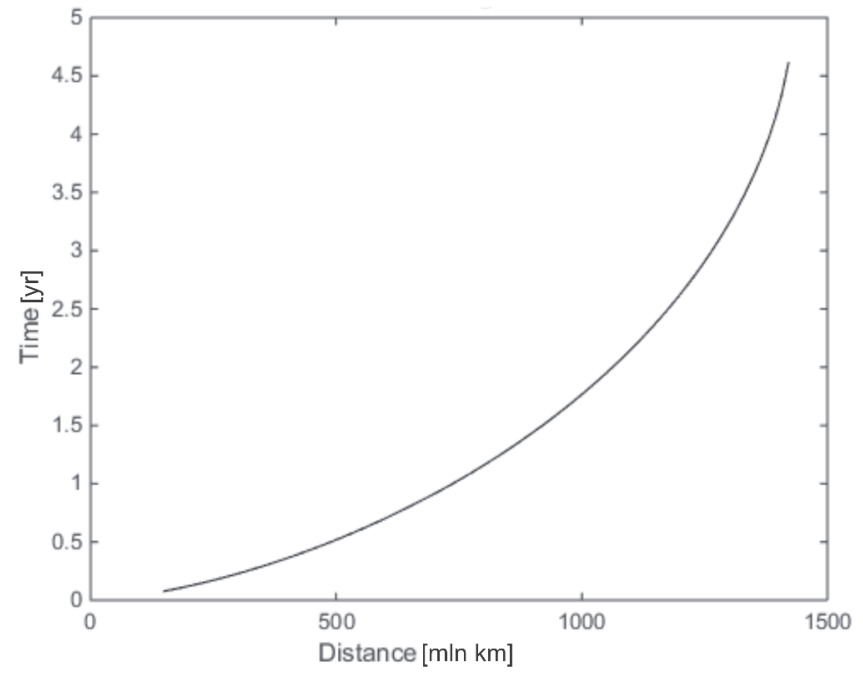

Fig. 7. Time of reaching the orbit of the Earth from a given distance from the Sun

This differential equation can be solved using the transformation:

$$
\sqrt{\frac{R_{0}}{2 G M}\left(\frac{r}{r-R_{0}}\right)} d r=d t
$$

After integration we get:

$$
\begin{gathered}
\sqrt{\frac{R_{0}}{2 G M}} \int \sqrt{\frac{r}{r-R}} d r= \\
=\sqrt{\frac{R_{0}}{2 G M}} \frac{\left(\sqrt{r}(r-R)+r \operatorname{atan}\left(\frac{\sqrt{r}}{\sqrt{R-r}}\right) \sqrt{R-r}\right)}{\sqrt{R-r}}=t
\end{gathered}
$$

The time of reaching the Earth's orbit versus the initial distance from the Sun is given in Figure 7 . The final velocity of grains falling from the Saturnian orbit to the orbit of the Earth is $\sim 40 \mathrm{~km} \mathrm{~s}^{-1}$.

During this time, depending on the size, chemical composition and albedo the grain may lose part of its mass. High albedo and low thermal conductivity resulting from the porosity could slow this mass loss. For successful transport it is only necessary to keep the mass sufficient to protect microorganisms from cosmic radiation.

A comet moving from the Kuiper Belt has a significant velocity crossing the orbit of Saturn. If the trajectory of this comet crosses also the orbit of the Earth it would be a faster means of transport than the free-falling boulders of initial low velocity considered above.

\section{DECELERATION IN THE ATMOSPHERE}

The physics of meteoroid entry into the atmosphere is discussed in many papers, e.g. Romig (1964), Brown et al. (2004), Trigo-Rodríguez et al. (2015). The average speed of orbital motion of the Earth is $29.78 \mathrm{~km} / \mathrm{s}$. This means that meteoroids falling from orbit of the Saturn could enter the terrestrial atmo- 
sphere with a speed in the range $\sim 11-70 \mathrm{~km} \mathrm{~s}^{-1}$. This corresponds to a range of energy (per $1 \mathrm{~kg}$ of the meteoroid's mass) from $5 \times 10^{7} \mathrm{~J} \mathrm{~kg}^{-1}$ to $2.5 \times 10^{9} \mathrm{~J} \mathrm{~kg}^{-1}$. The specific heat of ice and water are: $c_{\text {ice }}=1960 \mathrm{~J} \mathrm{~K}^{-1} \mathrm{~kg}^{-1}$ and $\mathrm{c}_{\text {water }}=4220 \mathrm{~J} \mathrm{~K}^{-1} \mathrm{~kg}^{-1}$, respectively. The heat of sublimation is $\mathrm{C}_{\text {sub }}=2.8310^{6} \mathrm{~J} \mathrm{~kg}^{-1}$. This means that even the lowest value of kinetic energy is large enough to increase the temperature of the meteoroid by a few hundred degrees Kelvin and enough for sublimation and/or vapourization of the whole mass of the meteoroid. Fortunately, the meteoroid moving in the atmosphere loses its energy by transferring it to the surrounding gas and by radiation. The effectiveness of deceleration due to radiation depends on the ratio surface/mass. For grains smaller than $10-100 \mu \mathrm{m}$ this ratio is high and such micrometeoroids could be decelerated in the upper atmosphere and possible microorganisms would be not heated to high temperatures (see also Wainwright et al., 2014).

A larger meteoroid could lose a substantial part of its mass. During deceleration of this body the dissipation of heat could be high, but initial low temperature, effect of ablation (i.e. sublimation of the surface; e.g., Campbell-Brown and Koschny, 2004) and short duration of the supersonic flight keep the interior of meteoroids cold. In fact, the temperature of interior of most meteorites is $\sim 0^{\circ} \mathrm{C}$ directly after falling - e.g., Pater and Lissauer (2001: 315-316). The final impact of the meteoroids on the planet surface will probably result in fragmentation of fragile icy meteorites but it need not be fatal for possible organisms in the their interior.

The comet is heated by Sun's radiation leading to sublimation of the volatiles and to other forms of activity, such as jets. The small grains (from the E-ring) transported within comets could be released close to the Earth, as a result of this activity. If grains enter the Earth's atmosphere in a brief time then the microorganisms may also reach the surface alive.

\section{DISCUSSION AND CONCLUSIONS}

A few scenarios of transport of some living organisms from the core of Enceladus to terrestrial planets are possible.

Scenario of transport of organisms in large grains (boulders):

1. A large impact ejects boulders with organisms from Enceladus with a velocity exceeding $5 \mathrm{~km} \mathrm{~s}^{-1}$. The ejected bodies could be from the surface or from some depth depending on the energy of the impact.

2. Depending on the direction of the velocity, some boulders may leave the Saturnian system and enter the trajectory towards terrestrial planets.

3. The size, chemical composition and albedo allow the survival of the bodies and protect any contained microorganisms from cosmic rays.

4. After entering the Earth's atmosphere, the bodies protect the organisms from high temperatures during braking.

Instead of steps 1 and 2, the following processes may be possible:

1. Jets of cryovolcanic activity eject large grains with organisms to the E-ring. Interaction of grains from the E-ring with the satellites of Saturn in the form of gravity assistance leads to acceleration of some grains up to the escape velocity of system of Saturn.

2. The escaped grains with small velocity with respect to the Sun fall towards the Sun and terrestrial planets.

Steps 3 and 4 are the same as for the scenario above.

Another scenario is appropriate for small grains:

1. Jets of cryovolcanic activity eject grains with organisms to the E-ring. The grains must save organisms for some time against radiation.

2. A comet crossing the E-ring gathers fresh material from the ring. Some grains are hidden in fractures and pores of the comet where organisms are protected against cosmic radiation. Note that the total area of the E-ring (the ring extends from $180,000 \mathrm{~km}$ to $480,000 \mathrm{~km}$ from the center of Saturn) is $0.62210^{18} \mathrm{~m}^{2}$, i.e. half a million times larger than than the cross-sections of Jupiter or Saturn.

3. Comets transport grains closer to the Sun.

4. At the distance of 1-2 AU from the Sun the comet becomes active. Cometary jets release the grains from the E-ring close to the Earth. They enter its atmosphere. The small size allows them safe deceleration and reaching the surface of the Earth.

The results outlined above indicate that it is possible to transfer safely living organisms from the core of Enceladus to other Solar System bodies. This transport is possible due to well-known and currently observed processes such as cryovolcanic activity, close passages of comets, gravitational assistance and/or meteorite impacts. For other bodies where presumed life could exist, similar transport requires more dramatic and less likely phenomena. For Earth and Venus only catastrophic collisions can release boulders. The Martian meteorites come from smaller but still large craters. For example, the SNC meteorites were probably ejected as a result of the impact that resulted in a crater more than $100 \mathrm{~km}$ across (Vickery and Melosh, 1987)

The possibility of picking organisms from the upper atmosphere of the Earth by comet or asteroid seems be negligible. This requires penetration of the atmosphere down to $\sim 70 \mathrm{~km}$ above the surface. At this altitude the atmosphere is dense and the fast-moving body is subject to intense heating at its surface. A large temperature reduces additionally the possibility of living organisms surviving. For a slow body, deep penetration of atmosphere leads rather to falling on to the Earth.

Within Europa, the satellite of Jupiter, life could exist in the global subsurface ocean. In this respect, the situation is similar to that of Enceladus. However, the escape velocity from the surface of Europa is significantly higher $\left(\sim 2 \mathrm{~km} \mathrm{~s}^{-1}\right)$. Moreover, for leaving Jupiter's system, the boulder needs additionally a velocity increase of $\sim 6 \mathrm{~km} \mathrm{~s}^{-1}$. So, to leave the system, the ejecta require a speed of $\sim 8 \mathrm{~km} \mathrm{~s}^{-1}$, which is about twice the energy needed to leave the surface of Enceladus. However, as for the grains from Europa, gravity assistance (there are 4 large satellites of Jupiter) could serve as a mechanism for ejecting boulders beyond the Jupiter system. Similar dynamical problems may be found for possible Mercurian meteorites, see Gladman and Coffey (2008).

Comparing dynamical constraints for panspermia from Enceladus and from other bodies one can conclude that 
Enceladus is the best candidate as a cradle of the life in the Solar System. Relatively simple investigation of jets from Enceladus could give an answer to the question of possible life inside Enceladus. Dr. Ch. McKey, a reviewer of the manuscript, stated that if the prediction of this paper was correct then a mission to the plume of Enceladus will discover LUCA perhaps with a few $\mathrm{Ga}$ of genetic drift (e.g., Steel et al., 2017).

The practical sense of panspermia is difficult to determine. It may be just a theory without any application. Biological evidences against the panspermia theory is discussed by Di Giulio (2010). On the other hand, there remain the controversial sug- gestions of Hoyle and Wickramasinghe (1978) that inflow of extraterrestrial genes is still continuing to enter the terrestrial atmosphere and that this could be an important factor for our health and evolution.

Acknowledgements. I am very grateful to the reviewers: Dr. Ch. McKay (Planetary Systems Branch), Dr. Xu Cai (Leiden University), and to an anonymous reviewer for their remarks and suggestions. Computer resources of the Interdisciplinary Centre for Mathematical and Computational Modeling of the University of Warsaw were also used in the research.

\section{REFERENCES}

Abramov, O., Mojzsis, S.J., 2011. Abodes for life in carbonaceous asteroids? Icarus, 213: 273-279.

Arrhenius, S., 1908. Worlds in the Making: The Evolution of the Universe. New York, Harper \& Row.

Batygin, K., Brown, M.E., 2010. Early dynamical evolution of the Solar System: pinning down the initial conditions of the Nice Model. The Astrophysical Journal, 716: 1323-1331.

Borg, L., Connelly, J.N., Nyquist, L.E., Shih Chi-Y., Wiesmann, H., Reese, Y., 1999. The age of the carbonates in Martian Meteorite ALH84001. Science, 286: 90-94.

Brasier, M.D., 2012. Secret Chambers: The Inside Story of Cells and Complex Life. Oxford University Press.

Brown, P., Pack, D., Edwards, W.N., Revelle, D.O., Yoo, B.B., Spalding, R.E., Tagliaferri, E., 2004. The orbit, atmospheric dynamics, and initial mass of the Park Forest meteorite. Meteoritics and Planetary Science, 39: 1781-1796.

Bryce, C.C., Horneck, G., Rabbow, E., Edwards, H.G.M., Cockell, Ch.S., 2014. Impact shocked rocks as protective habitats on an anoxic early Earth. International Journal of Astrobiology, 14: 115-122.

Campbell-Brown, M.D., Koschny, D., 2004. Model of the ablation of faint meteors. A\&A, 418: 751-758.

Clarke, A., 2014. The thermal limits to life on Earth. International Journal of Astrobiology, 13: 141-154.

Ćuk, M., Dones, L., Nesvorný, D., 2016. Dynamical evidence for a late formation of Saturn's Moons. The Astrophysical Journal, 820, article ID 97: 1-16; doi: 10.3847/0004-637X/820/2/97

Czechowski, L., 2014a. Some remarks on the early evolution of Enceladus. Planetary Space Science, 104: 185-199.

Czechowski, L., 2014b. Enceladus: a cradle of life of the Solar System? Geophysical Research Abstracts, 16: EGU2014-9492-1.

Czechowski, L., Losiak, A., 2016. Early thermal history of Rhea: the role of serpentinization and liquid state convection. Acta Geophysica, 64: 2677-2716.

Di Giulio, M., 2010. Biological evidence against the panspermia theory. Journal of Theoretical Biology, 266: 569-572.

Dodd, M.S., Papineau, D., Grenne, T., Slack, J.F., Rittner, M., Pirajno, F., O'Neil, J., Little, C.T.S., 2017. Evidence for early life in Earth's oldest hydrothermal vent precipitates. Nature, 543: $60-64$.

Dunham, W., 2017. Canadian bacteria-like fossils called oldest evidence of life. Reuters. Retrieved 1 March 2017, http://ca.reuters.com/article/topNews/idCAKBN16858B?sp=true

Gladman, B., Coffey, J., 2008. Mercurian impact ejecta: meteorites and mantle. Asteroids, Comets, Meteors, 8289.pdf

Góbi, S., Kereszturi, A., 2017. Role of serpentinization in the thermal and connected mineral evolution of planetesimals - evaluating possible consequences for exoplanetary systems. Monthly Notices of the Royal Astronomical Society, 466: 2099-2110.
Guess, A.W., 1962. Poynting-Robertson effect for a spherical source of radiation. Astrophysical Journal, 135: 855-866.

Hargitai, H., Kereszturi, A., eds., 2015. Encyclopedia of Planetary Landforms. Springer, New York, ISBN 1461431336.

Hoyle, F., Wickramasinghe, C., 1978. Lifecloud - The Origin of Life in the Universe. Harper \& Row. ISBN-10: 0060119543.

Hsu, H.-W., Postberg, F., Sekine, Y., Shibuya, T., Kempf, S., Horanyi, M., Juhasz, A., Altobelli, N., Suzuki, K., Masaki, Y., Kuwatani, T., Tachibana, S., Sirono, S.I., MoragasKlostermeyer, G., Srama, R., 2015. Ongoing hydrothermal activities within Enceladus. Nature, 519: 207-210.

Izawa, M.R.M., Banerjee, N.R., Flemming, R.L., 2010. Basaltic glass as a habitat for microbial life: implications for astrobiology and planetary exploration. Planetary Space Science, 58: 583-591.

Kohler, S., 2015. Geysers from the Tiger Stripes of Enceladus. Astronomy Abstract Services, AAS Nova Highlight, 02 Oct 2015, id.336.

Malamud, U., Prialnik, D., 2013. Modeling serpentinization: applied to the early evolution of Enceladus and Mimas. Icarus, 225: 763-774.

McKay, C.P., 2016. Titan as the abode of life. Life, 6: 8; doi:10.3390/life6010008; http://www.mdpi.com/2075-1729/6/1/ 8/htm, retrieved 24.Oct.2017.

McKay, D.S., Gibson, E.K. Jr., Thomas-Keprta, K.L., Vali, H., Romanek, Ch.R., Clemett, S.J., Chillier, X.D.F., Maechling, C.R., Zare, R.N., 1996. Search for past life on Mars: possible relic biogenic activity in Martian Meteorite ALH84001. Science, 273: 924-930.

Melosh, H.J., 2011. Planetary Surface Processes. Cambridge University Press

Mousis, O., Lunine, J.I., Waite, J.H. Jr., Magee, B., Lewis, W.S., Mandt, K.E., Marquer, D., Cordier, D., 2009. Formation conditions of Enceladus and origin of its methane reservoir. The Astrophysical Journal, 701: L39-L42.

Nimmo, F., 2004. What is the young's modulus of ice? Europa's Icy Shell (2004) 7005 pdf; http://www.lpi.usra.edu/meetings/europa2004/pdf/7005.pdf

Nitschke, W., Russell, M.J., 2010. Just Like the universe the emergence of life had high enthalpy and low entropy beginnings. Journal of Cosmology, 10: 3200-3216.

O'Leary, M., 2008. Anaxagoras and the Origin of Panspermia Theory. Universe Publishing Group. ISBN 978-0-595-49596-2.

Pabst, W., Gregorová, E., 2013. Elastic properties of silica polymorphs - a review. Ceramics - silikáty, 57: 167-184.

Pater, de, I., Lissauer, J.J., 2001. Planetary Sciences. Cambridge University Press.

Porco, C.C., Helfenstein, P., Thomas, P.C., Ingersoll, A.P., Wisdom, J., West, R., Neukum, G., Denk, T., Wagner, R., 
Roatsch, T., Kieffer, S., Turtle, E., McEwen, A., Johnson, T.V., Rathbun, J., Veverka, J., Wilson, D., Perry, J., Spitale, J., Brahic, A., Burns, J.A., Del Genio, A.D., Dones, L., Murray, C.D., Squyres, S., 2006. Cassini observes the Active South Pole of Enceladus. Science, 311: 1393-1401.

Romig, M.F., 1964. The physics of meteor entry. The RAND Corporation, Santa Monica, California. Retrieved 24 Oct. 2017, https://www.rand.org/content/dam/rand/pubs/papers/2008/P2902.pdf

Russell, M.J., Hall, A.J., Martin, W., 2010. Serpentinization as a source of energy at the origin of life. Geobiology, 8: 355-371.

Schubert, G., Anderson, J.D., Travis, B.J., Palguta, J., 2007. Enceladus: present internal structure and differentiation by early and long-term radiogenic heating. Icarus, 188: 345-355.

Sharov, A.A., Gordon, R., 2013. Life Before Earth, arXiv:1304.3381 [physics.gen-ph]. https://arxiv.org/ftp/arxiv/papers/1304/1304.3381.pdf

Steel, E.L., Davila, A., McKay, C.P., 2017. Abiotic and biotic formation of amino acids in the Enceladus Ocean. Astrobiology, 17: 862-875.

Trigo-Rodríguez, J.M., Lyytinen, E., Gritsevich, M., Morenolbáñez, M., Bottke, W.F., Williams, I., Lupovka, V., Dmitriev, V., Kohout, T., Grokhovsky, V., 2015. Orbit and dynamic origin of the recently recovered Annama's H5 chondrite. Monthly Notices of the Royal Astronomical Society, 449: 2119-2127.
Thomson, W., 1871. Presidential Address to the British Association for the Advancement of Science, assembled at Edinburgh, 1871, printed in London.

Vickery, A.M., Melosh, H.J., 1987. The large crater origin of SNC Meteorites. Science, 237: 738-743.

Wainwright, M., Wickramasinghe, N.C., Rose, C.E., Baker, A.J., 2014. Recovery of cometary microorganisms from the stratosphere. Journal of Astrobiology and Outreach, 2: 110; doi:10.4172/2332-2519.1000110

Waite, J.H. Jr., Lewis, W.S., Magee, B.A., Lunine, J.I., McKinnon, W.B., Glein, C.R., Mousis, O., Young, D.T., Brockwell, T., Westlake, J., Nguyen, M.-J., Teolis, B.D., Niemann, H.B., McNutt, R.L., Perry, M., Ip, W.-H., 2009. Liquid water on Enceladus from observations of ammonia and ${ }^{40} \mathrm{Ar}$ in the plume. Nature, 460: 487-490.

Weiss, M.C., Sousa, F.L., Mrnjavac, N., Neukirchen, S., Roettger, M., Nelson-Sathi, S., Martin, W.F., 2016. The physiology and habitat of the last universal common ancestor. Nature Microbiology, 1, article number: 16116; doi:10.1038/nmicrobiol.2016.116

Yang, Y., Yokobori, S., Yamagishi, A., 2009. Assessing panspermia hypothesis by microorganisms collected from the high altitude atmosphere. Biological Sciences in Space, 23: 151-163. 